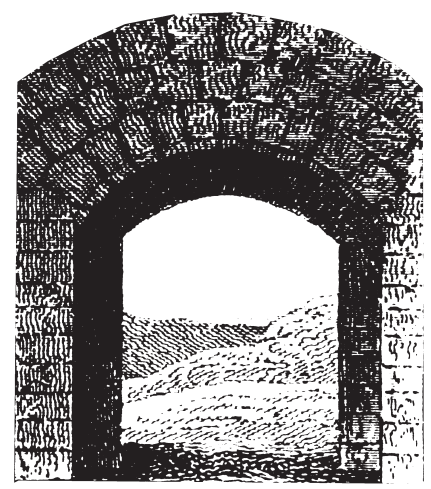

\title{
INFORMACIÓN BIBLIOGRÁFICA Y NOTICIAS
}

\section{RESEÑAS}

\section{Maestras contemporáneas Jové, G. \\ Edicions de la Universitat de Lleida, Lleida, 2017, 182 pp.}

«Maestras contemporáneas», un título corto y conciso pero que evidencia el claro protagonismo que toma en esta obra la figura femenina en el ámbito educativo. Como dice la misma autora, se centra en las maestras porque más del $70 \%$ de las profesionales del ámbito de la educación son mujeres, y la presencia de estas también se expande en muchos otros contextos educativos.

Bajo este título, empieza cuestionándose ciertos aspectos relativos al mundo actual de la educación: sus modelos docentes, sus resistencias u obstáculos ante el cambio, de qué forma podemos plantearnos acciones para este en pleno siglo XXI... Sin duda es una obra de reflexión, pero también de propuestas de acción y de nuevos retos que se comparten con las lectoras y los lectores del libro.
Preguntas como ¿Por qué en el mundo de la educación se habla tanto de la necesidad del cambio y cuesta tanto su implementación y sobretodo su continuidad? ¿Por qué están tan arraigados algunos modelos docentes? ¿Por qué cuesta tanto modificarlos? Y, por tanto, ¿de qué manera podemos articular los espacios formativos para deconstruir y reconstruir estos modelos que tenemos tan interiorizados? ¿De qué manera podemos pasar a la acción?

Estos son los principales interrogantes que la autora de esta obra se ha planteado y a partir de los cuales ha desarrollado un doble discurso presentado en el libro mediante diversas experiencias (capítulos). Por una parte, un discurso de reflexión sobre la necesidad de un cambio en el sistema educativo, en los currículums educativos, en los libros de texto, en las aulas y en las mentes de maestras y maestros resistentes al cambio. Y, por otro lado, un discurso de acción mediante propuestas y proyectos educativos en los que el arte contemporáneo y el proceso creativo son dos elementos clave para cuestionarse la profesión de maestras, lo que acontece en las aulas, y lo que sucede fuera de ellas, que es donde trascurre realmente la vida.

Algunas de estas experiencias o vivencias que la autora comparte con las lectoras y los lectores del libro giran en torno a ideas tales como:

- La educación con, entre, y para con todos (educación colaborativa, de cooperación, de reciprocidad, de compañía, etc.). - El compromiso social de las maestras, de las presentes y de las futuras que se están formando. Un compromiso desde la ética con los niños y las niñas, con el territorio, con el contexto, y con la comunidad. - La necesidad de construir puentes entre el arte y la educación. Los museos como laboratorios de aprendizaje, como espacios educativos para alumnado y para maestras que buscan nuevos lugares para aprender, crear y reflexionar sobre lo co- 
tidiano de la educación y de la vida. Espacios de encuentro y diálogo con el arte, artistas contemporáneos, con sus procesos creativos y sus mensajes reflexivos para aprender a observar y a entender la sociedad actual.

- La importancia de repensar el papel de los libros de texto y su uso en las escuelas, desconectados de la realidad, de las vidas presentes de las maestras y las estudiantes, y de los niños y niñas.

- Los objetos hablan. Los objetos permiten aprender sobre el paso del tiempo, las costumbres, la vida pasada y presente, sobre materiales, sobre tradiciones, sentimientos y emociones.

Contenidos, todos ellos, que se nutren de las experiencias, vivencias, intercambios y lecturas en las que la autora se ha visto involucrada a lo largo de sus «viajes» de aprendizaje y reflexión, y que se traducen en propuestas concretas de acción para el cambio, para repensar el currículum y las formas y modelos de educar, en general, y en particular, en el ámbito educativo artístico contemporáneo.

Esta obra, pues, no solo invita a sus lectoras y lectores a cuestionarse cómo estamos educando y con qué modelos en pleno siglo XXI, sino que, con sus propuestas creativas, artísticas y de acción para el cambio en el ámbito educativo, la autora también les brinda la oportunidad de crear nuevos procesos posibles y mudanzas educativas.

Dra. Laia Coma Quintana Universitat de Barcelona

\section{Materiales didácticos para la formación ciudadana $^{\mathrm{i}}$}

En esta reseña se analizan cinco unidades didácticas nacidas del proyecto Desarrollo de la formación sociopolítica para una ciudadanía democrática: diseño e implementación de materiales didácticos en Ciencias Sociales. (EDU2015-65621-C33-R), publicadas en Barcelona en 2017.

Cada una de estas unidades busca formar al estudiante de ESO en diversas competencias estructurantes, como la social ciudadana o la digital, entre otras. Como exponen Fuentes, Sabido-Codina y Albert (2017), todas ellas se caracterizan por ser propias de la disciplina, usar el método científico de la historia, ser interdisciplinares y estar adecuadas curricularmente. Asimismo, buscan un aprendizaje activo, a partir de eviden- cias históricas y de un trabajo, tanto individual, como grupal.

La primera, Atenes: $:^{i i}$ L'origen de la democràcia, es una unidad didáctica diseñada para llevar a cabo en $1^{\circ}$ de la ESO, programada para: potenciar la identificación de las formas de participación de la ciudadanía dentro del sistema democrático; la distinción entre dictadura, democracia directa y democracia representativa; la comparación entre el sistema democrático, desde su contexto de origen (Atenas), y el sistema político español, actual; y el análisis e interpretación de fuentes históricas, como método de aprendizaje.

Para su aplicación, se prevén once sesiones en las que las actividades de aprendizaje se organizan en cuatro bloques que conducen al alumnado a comprender progresivamente, y mediante la empatía histórica: las formas de participación ciudadana, la formación, el funcionamiento y los límites de la democracia ateniense y de sus instituciones, y la evolución del sistema democrático hasta el presente, finalizando con el análisis del sistema español.

Dichas actividades parten de fuentes históricas, a través del análisis de las cuales, el alumnado puede reflexionar sobre las condiciones y situación en que se desarro1la la democracia, para comprender el término con mayor profundidad (Prats, Fuentes y Sabariego, 2019).

En cuanto a la evaluación, los autores contemplan un conjunto de instrumentos a partir de los cuales se plantea, tanto el seguimiento de los aprendizajes por parte del docente, como la autoevaluación y reflexión metacognitiva de los propios alumnos.

La segunda, Els amors de la meva vida, ${ }^{i i i}$ es un conjunto de actividades individuales, diseñadas para llevar a cabo en $3^{\circ}$ de la ESO, así como para ser adaptadas a otros niveles educativos. Las autoras nos plantean la necesidad de reconocer y analizar nuestra concepción del amor en el sentido más amplio de la palabra. El objetivo principal es poner en duda todo lo que sabemos sobre este, con la intención de reflexionar alrededor de los mitos que hemos normalizado dentro de nuestra sociedad patriarcal y heteronormativa.

Para llevarlo a cabo, se prevén diez sesiones, en las que se plantean seis actividades. La primera consiste en identificar los propios sentimientos hacia las personas amadas, la intensidad y los motivos. La segunda se enfoca a la reflexión sobre el papel de la publicidad en la creación de estereotipos y la promoción de la violencia de género. La tercera aborda los conflictos que se establecen en situaciones de abuso y control de la pareja, y medidas como el empoderamiento y la autoestima, para combatir dichas circunstancias. La cuarta consiste en reflexionar sobre las prácticas y modelos de relaciones afectivas que conocen, para tomar consciencia de la salubridad de cada caso. La quinta motiva a los alumnos a diseñar y elaborar una estrategia con la que motivar un cambio en los modelos de relación, hacia una dirección saludable. Y, por último, la sexta se plantea como un espacio en el que reflexionar sobre lo aprendido.

Las actividades contemplan una metodología que requiere la observación y creación de contenido audiovisual, y una actitud de diálogo y trabajo cooperativo entre iguales. Además, al finalizar cada una de las sesiones, se incluye la propuesta de una reflexión individual.

En cuanto a la evaluación, tres de las seis sesiones incluyen unas rúbricas en las que se contemplan ítems relacionados con el interés y la participación de los miembros de cada grupo, así como con el desarrollo de la tarea, según las indicaciones iniciales de las actividades.

La tercera, The maze, ${ }^{i v}$ nosotros y ellos, es una unidad didáctica diseñada para llevar a cabo en $4^{\circ}$ de la ESO, con el objetivo de trabajar los conceptos de identidad y diferencia, para abordar aspectos como: la construcción del nosotros y la asignación del papel de un ellos, los derechos humanos, la esclavitud, los prejuicios y la discriminación.

Para llevarlo a cabo, sus creadores nos presentan tres casos históricamente significativos. En primer lugar, las identidades y concepciones de los bárbaros y los romanos. Seguidamente, las de los arios, como pasado menos lejano. Y en último lugar, para referir lo que podría llegar a pasar en un futuro, exponen distopías a las que podríamos llegar desde la sociedad actual, en caso de llevar algunas de nuestras actitudes presentes al extremo.

Los autores plantean el uso de la gamificación como estrategia metodológica. Así pues, uno de los aspectos más interesantes de esta unidad didáctica es la propuesta del uso de juegos de rol, como por ejemplo ClassCraft, para crear un avatar al que asignar los puntos que van obteniendo con la realización de las tareas. 
En relación a esto último, las actividades que podemos encontrar tras cada uno de los bloques recién referenciados, se clasifican en Side Quest y Main Quest. Todas ellas enfocadas a la reflexión mediante textos, vídeos o representaciones sobre los determinados temas. La diferencia es que, mientras que las primeras son de carácter obligatorio, y necesarias para su evaluación, las otras son de carácter voluntario, para profundizar en los aprendizajes, y obtener ventajas como, por ejemplo, tiempo extra a la hora de realizar cualquier otra tarea.

La cuarta, Dones i Revolució Industrial, ${ }^{v}$ está diseñada para llevar a cabo en $4^{\circ}$ de la ESO. Con ella se pretende potenciar la comprensión de las causas y consecuencias de la revolución industrial, mediante el método del historiador; analizar las revoluciones paralelas, e incorporando la visibilización del papel de las mujeres en dicho contexto.

Para ello, se plantean once sesiones de trabajo y una salida final. En la primera sesión, se contempla la visualización de un documental para introducir los principales temas de estudio. Las cuatro siguientes consisten en preparar y elaborar, por grupos, exposiciones para presentar las revoluciones paralelas. La sexta consiste en leer sobre los sindicatos y su aparición, y en preparar un guion para entrevistar a los representantes sindicales. En la séptima, se prevé la conferencia de estos invitados a la escuela, para hacer una exposición de su ocupación. Las dos siguientes se enfocan a analizar los contenidos trabajados a través de una película. Y las últimas se enfocan a la elaboración de un itinerario, a través de un geolocalizador como My Maps, en el que cada grupo desarrolla uno de los puntos de interés y en llevar a cabo una exposición in situ del trabajo realizado.

En cuanto a la metodología de trabajo, la propuesta se organiza a través de un hilo conductor - proyecto del ayuntamiento para la recuperación y difusión de la memoria histórica- enfocado a darle valor a la tarea que se va a desarrollar. Para llevarla a cabo, se organiza al alumnado en grupos, y se presta el acompañamiento necesario para la dinámica del trabajo cooperativo.

Para evaluar a los grupos de trabajo, el material incluye tres tipos de rúbricas en las que se contemplan ítems relacionados con el desarrollo de la tarea, sus habilidades comunicativas y de búsqueda de información, sus aptitudes y su organización, entre otros.
La quinta, Totalitarismes $i$ democràcies, ${ }^{v i}$ es una propuesta de trabajo interdisciplinario diseñada para llevar a cabo en $4^{\circ}$ de la ESO. Los objetivos principales de este material son conocer y analizar los procesos más relevantes de la historia y la economía del período entre guerras, la Segunda Guerra Mundial y sus consecuencias; fomentar la participación en la vida democrática y conocer la diferencia entre regímenes totalitarios y democráticos.

Para ello, se prevé una dedicación de tres horas semanales durante un mes y medio. En este tiempo se trabaja mediante cinco pequeñas guías con: información sobre cómo proceder, fuentes históricas para comprender los marcos políticos, actividades de investigación y de síntesis, y recursos de consulta en línea. La primera guía invita a los alumnos a elaborar un árbol genealógico que abarque sus cuatro últimas generaciones, la segunda a identificar los regímenes políticos que había en el país de cada una de estas, la tercera a investigar y exponer sobre el nazismo, fascismo, estalinismo y franquismo; la cuarta a investigar y exponer las características de los estados europeos, y la quinta a elaborar una infografía para sintetizar los aprendizajes realizados.

En cuanto a la metodología de trabajo, la unidad didáctica se organiza a través de la propia curiosidad, surgida de la elaboración de los árboles genealógicos del alumnado. En general, se contempla el trabajo en grupos cooperativos y destaca el uso de las herramientas TIC, en todos los apartados.

Respecto a la evaluación, la propuesta considera tres tipos de evaluación. La inicial, mediante un portafolio digital, individual. La continuada, mediante el portafolio y rúbricas de seguimiento. Y la final, a través de los instrumentos anteriores, más una infografía.

Con todo esto, podemos decir que el diseño de estos materiales motiva a los alumnos a pensar, indagar y descubrir, de manera autónoma, para dar respuesta a las diversas cuestiones que se les van planteando. Y, por lo tanto, que prioriza que el alumnado tenga un papel protagonista en su formación. Además, favorece el aprendizaje significativo y la construcción de los esquemas de conocimiento, mediante un trabajo interdisciplinario y conectado a la realidad cercana de los estudiantes. En relación a esto, cabe destacar que las programaciones no solo promueven la adquisición de las competencias propias de las ciencias sociales, sino también habilida- des comunicativas, competencia digital y consciencia crítica, para promover un papel activo y responsable en la construcción de la sociedad en la que conviven.

\section{NOTAS}

${ }^{\mathrm{i}}$ Acceso a los materiales desde la web del grupo de investigación DHiGeCs: www.ub.edu/ dhigecs

ii BELLATTI, I., FERNÁNDEZ, E. y NARVÁEZ, M. (2017). Atenes: L'origen de la democràcia. Barcelona: Aguiló Gràfic SL. Programa Estatal I+D+i Orientat als reptes de la Societat EDU2015-65621-C3-3-R «Desarrollo de la formación sociopolítica para una ciudadanía democrática: diseño e implementación de materiales didácticos en Ciencias Sociales». Investigador Principal del proyecto: Joaquim Prats Cuevas. Financiado por: Ministerio de Economía y Competitividad. Depósito legal: B 22501-2017.

iii AZNAR, R. M., BARRIGA, E., CANDELA, A. y CHAMIZO, M. (2017). Els amors de la meva vida. Barcelona: Aguiló Gràfic SL. Programa Estatal I+D+i Orientat als reptes de la Societat EDU2015-65621-C3-3-R «Desarrollo de la formación sociopolítica para una ciudadanía democrática: diseño e implementación de materiales didácticos en Ciencias Sociales». Investigador Principal del proyecto: Joaquim Prats Cuevas. Financiado por: Ministerio de Economía y Competitividad. Depósito legal: B 22501-2017.

iv MEDINA, D., HURTADO, D., MOLINA, J., SABIDO-CODINA, J., y GIL, N. (2017). The Maze, nosotros y ellos. Barcelona: Aguiló Gràfic SL. Programa Estatal I+D+i Orientat als reptes de la Societat EDU2015-65621-C33-R «Desarrollo de la formación sociopolítica para una ciudadanía democrática: diseño e implementación de materiales didácticos en Ciencias Sociales». Investigador Principal del proyecto: Joaquim Prats Cuevas. Financiado por: Ministerio de Economía y Competitividad. Depósito legal: B 22501-2017.

v AMATLLER, A., BORCH, D., IZCARA, C., FUENTES, C., SÁEZ, I. y VICENTE, S. (2017). Dones i Revolució Industrial. Barcelona: Aguiló Gràfic SL. Programa Estatal I+D+i Orientat als reptes de la Socetat EDU201565621-C3-3-R «Desarrollo de la formación sociopolítica para una ciudadanía democrática: diseño e implementación de materiales didácticos en Ciencias Sociales». Investigador Principal del proyecto: Joaquim Prats Cuevas. Financiado por: Ministerio de Economía y Competitividad. Depósito legal: B 22501-2017. 
vi ALBERT, J. M., DE LA TORRE, J. L., LEÓN, S. y SÁNCHEZ, M. A. (2017). Totalitarismes i democràcies. Barcelona: Aguiló Gràfic SL. Programa Estatal I+D+i Orientat als reptes de la Socetat EDU2015-65621-C33-R «Desarrollo de la formación sociopolítica para una ciudadanía democrática: diseño e implementación de materiales didácticos en Ciencias Sociales». Investigador Principal del proyecto: Joaquim Prats Cuevas. Financiado por: Ministerio de Economía y Competitividad. Depósito legal: B 22501-2017.

\section{REFERENCIAS BIBLIOGRAFICAS:}

FUENTES, C., SABIDO, J., y ALBERT, J. M. (2017). Educación para la ciudadanía: experiencias didácticas. En R. LÓPEZ, P. MIRALLES, J. PRATS (dirs.) y C. J. GÓMEZ (coord.) (2017). Enseñanza de la historia y competencias educativas. (pp. 87-104). Graó: Barcelona.

PRATS, J., FUENTES, C., y SABARIEGO, M. (2019). La investigación evaluativa de ma- teriales didácticos para la educación política y ciudadana a través de contenidos históricos. Revista Electrónica Interuniversitaria de Formación del Profesorado, 22(2), 1-15. Recuperado de: http://www.ub.edu/histodidac tica/images/documentos/pdf/prats\%20Rei fop.pdf

Marina Sánchez Hostalot Universitat de Barcelona 